\title{
In Situ Measurements of the Complex Permittivity of Materials Using Reflection Ellipsometry in the Microwave Band: Theory (Part I)
}

\author{
Florence Sagnard, Faroudja Bentabet, and Christophe Vignat
}

\begin{abstract}
The aim of this series of two papers is to propose an original and low-cost tool dedicated to industrial applications and based on the reflection ellipsometry technique for in situ characterization of dielectric materials at microwave frequencies. In this first paper, different theoretical developments are presented that concern first a specific numerical method for calculating the complex permittivity of a single-layer sample from the measured parameters. Based on contour line charts, this method allows obtaining simultaneously the relative uncertainties on the real and imaginary parts of the complex permittivity. Secondly, for experimental comparisons with the classical Fresnel method, a numerical data processing method based on contour line charts has also been developed, which aims at the determination of the reflection coefficients in both parallel and perpendicular polarizations of the material.
\end{abstract}

Index Terms-Complex permittivity, ellipsometry, free-space methods, Fresnel coefficients, material characterization, microwave frequencies, polarization, reflexion.

\section{INTRODUCTION}

D IELECTRIC characterization of materials at radio and high frequencies has been intensively developed over a long period of time [1]. Among the implemented techniques, free-space methods are well suited for industrial applications [2], [3], such as civil engineering (moisture content evaluation, change in the material composition during time) [4]-[6], nondestructive testing, ground penetrating radar [7], and communication systems (modeling of radiowave propagation channels) [8]-[10]. They can be first distinguished by their measurement domain, either time or frequency, then by the type of the measured quantities, either scalar or vector valued, and also by the experimental configuration using reflection and/or transmission at a fixed or variable angle of incidence. The major advantages of these methods are their nondestructive characteristics, their suitability for use in broadband, and their averaging property due to the large footprint illuminating the sample. In order to estimate the dielectric parameters (the real and imaginary permittivities), several free-space experimental systems have been developed in the past years, particularly in the millimeter band [11]-[24]. These methods are based on the measurement of the reflection and/or transmission coefficients

Manuscript received May 11, 2004; revised September 13, 2004. This work was supported by the French Department of Research (ANVAR).

F. Sagnard is with the Université de Marne-La-Vallée, Cité Descartes, 77454 Marne-La-Vallée Cedex 02, France, and with INSA de Rennes, IETR, 35043 Rennes Cedex, France (e-mail: florence.sagnard@insa-rennes.fr).

F. Bentabet and C. Vignat are with the Université de Marne-La-Vallée, Cité Descartes, 77454 Marne-La-Vallée Cedex 02, France.

Digital Object Identifier 10.1109/TIM.2005.847203 associated with a flat large-aperture sample placed sufficiently far away from the transmitting antenna. They can be used in different configurations of angle of incidence, frequency, thickness, or polarization state. Their basic underlying principle is that the knowledge of the complex permittivity of an homogenizable material $\left(\tilde{\varepsilon}=\varepsilon^{\prime}-j \varepsilon^{\prime \prime}\right)$ allows, using the Fresnel formulas [25], to compute the reflection or transmission coefficients for an arbitrary angle of incidence $\theta_{i}$ (approach named here the Fresnel method) and different polarization states of the receiving antenna (the ellipsometry method) [26]. Thus, conversely, the complex permittivity of the material can be deduced back from the power of the reflected or/and transmitted part of the wave, as a function of the angle of incidence (Fresnel method) or of the polarization of the receiving antenna (reflection or transmission ellipsometry). In the proposed approach, only the reflection configuration is considered (assuming specular reflection, the angle of incidence equals the angle of reflection) and the two previous methods have been implemented by the authors on a unique measurement setup named COTREMO.

In this paper, our main contribution concerns the following areas:

1) development of reflection ellipsometry extended to the microwave domain (first proposed by Stetiu [27]): for decades, ellipsometry had been used only at optical submillimeter and millimeter frequencies [28]-[32];

2) design of a multistep algorithm for the processing of measurement data using graphical means (contour line charts) for the determination of the real and imaginary parts of the permittivity of a single layer sample (the thickness being assumed known) using the Fresnel and ellipsometry methods;

3) comparison of complex permittivity estimations obtained by both free-space techniques, the Fresnel method, and reflection ellipsometry (see part II of this paper [34]);

4) for each method, studies concerning the influence of relevant measurement parameters and of their uncertainties on the final estimation of the real and imaginary parts of the permittivity.

In fact, reflection ellipsometry in the microwave band appears as a promising technique for industrial applications because of its following advantages. First, the complex ratio of the reflection or transmission coefficients can be obtained using only scalar measurements; thus, the instrumentation is based on a low-cost detection system which relies on the use of a powermeter, an alternative and inexpensive choice to a vector network 


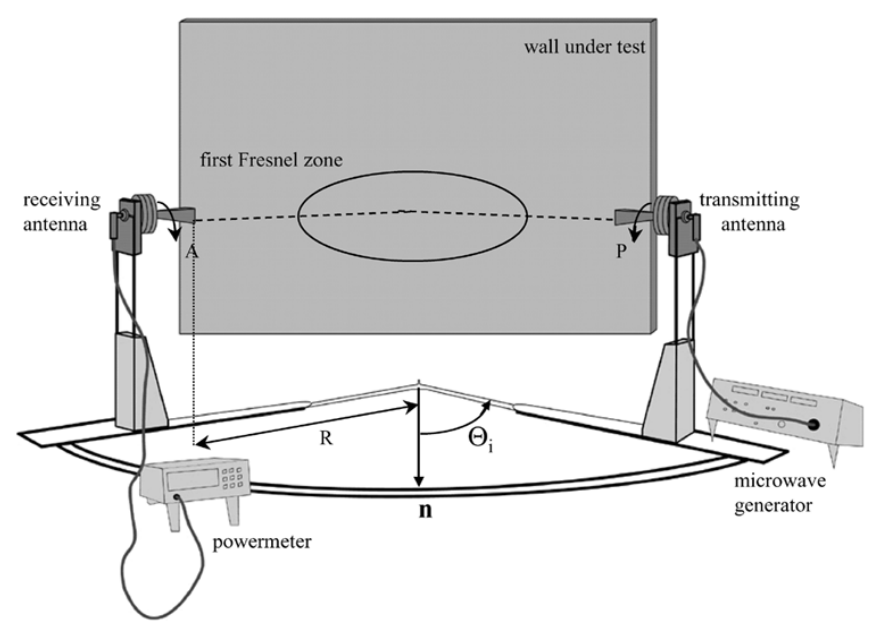

Fig. 1. Setup for the measurement of reflected waves.

analyzer. Secondly, contrary to the Fresnel method, ellipsometry requires only one fixed angle of incidence, so that the contribution of the line-of-sight wave path (weighted by the radiation pattern) to the overall reflected field received by the antenna can be minimized. At last, the sample thickness and the complex permittivity of a single-layer material can be theoretically determined from measurements at two angles of incidence; some developments about this feature are currently performed by the authors and have recently been published [33].

Section II presents the Fresnel method and its associated numerical developments. Section III describes the theoretical principles of reflection ellipsometry and its multistep numerical data processing. In Section IV, a parameter study is performed for the determination of optimal experimental conditions. Section V of this paper is dedicated to concluding remarks. Experimental results of complex permittivity estimations of common homogenizable materials (concrete, fiberboard, PVC, plasterboard) obtained by reflection ellipsometry in the microwave band are given in part II of this paper [34].

\section{FReSNEL Model}

\section{A. General Principles}

The Fresnel method consists in measuring the reflected power, in the cases of the parallel and perpendicular polarizations, as a function of the angle of incidence $\theta_{i}$ (see Fig. 1) [25], [26]. The complex permittivity can then be estimated using a nonlinear least squares method in which the theoretical expressions of the powers of either joint parallel (TM) and perpendicular (TE) reflection coefficients, or of their ratio, are fitted to the measured data [11], [17]-[20]. The theoretical curve that fits best the measured data yields the optimum estimate of the complex permittivity.

We assume, as a first-order approximation, that the material structure is made of multilayered homogeneous-or homogenizable ${ }^{1}$-media: thus, following the homogenization theory [35], it can be modeled by an isotropic homogeneous single-

${ }^{1}$ Inclusions are supposed very small compared to the wavelength inside the dielectric matrix.

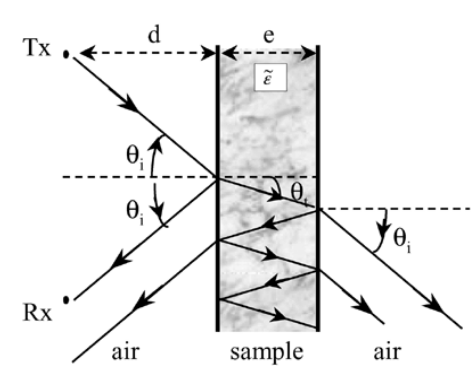

(a)

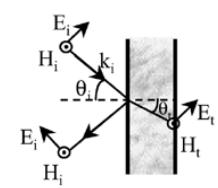

(b)

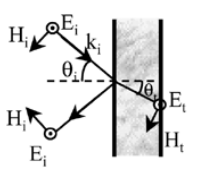

(c)
Fig. 2. Multiple reflections within a single-layer sample.

layer sample (with thickness denoted as $e$ ) with the same macroscopic electromagnetic response and a certain effective complex permittivity. It is assumed moreover that the transmitting antenna generates a quasi-plane wave front at his aperture and a linearly polarized electric field at the sample surface. In order to fulfill these requirements, we have chosen a pair of horn antennas characterized by a phase error significantly lower than $180^{\circ}$ at the aperture.

Fig. 2 shows the principle of multiple reflections within a single-layer isotropic dielectric sample, under the general assumptions expressed in the Introduction. The total reflected wave is constructed from the contribution of a large number of parallel rays reflected on and transmitted through the sample [25], [26]. Therefore, the resulting reflected field is proportional to the incident field

$$
\tilde{E}_{r}=\tilde{r}\left(F, \theta_{i}, \tilde{\varepsilon}_{r}\right) \tilde{E}_{i}
$$

The resulting complex reflection coefficient $\tilde{\mathbf{r}}$ is expressed as a function of frequency $F$, angle of incidence $\theta_{i}$, and relative complex permittivity $\tilde{\varepsilon}_{\mathrm{r}}$ in the following compact form [11], [26]:

$$
\tilde{r}\left(F, \theta_{i}, \tilde{\varepsilon}_{r}\right)=\frac{1-\exp (-j 2 \beta)}{1-\tilde{r}^{\prime 2} \exp (-j 2 \beta)} \tilde{r}^{\prime}
$$

where $\beta=k_{0} e \sqrt{\tilde{\varepsilon}_{r}-\sin ^{2} \theta_{i}}$ is the complex propagation factor through the sample slab, $k_{0}=(2 \pi) /\left(\lambda_{0}\right)$ is the free-space wave number, $\lambda_{0}$ is the free-space wavelength, $e$ is the thickness of the material, $\tilde{\varepsilon}_{r}=\varepsilon_{r}^{\prime}-j \varepsilon_{r}^{\prime \prime}$ is the relative complex permittivity of the dielectric sample, and $\tilde{r}^{\prime}$ is the reflection coefficient at the air-medium interface.

Choosing $\tilde{r}^{\prime}=\tilde{r}_{p}$ (respectively, $\tilde{r}^{\prime}=\tilde{r}_{s}$ ) in (2), the parallel (p) and perpendicular (s) $)^{2}$ complex reflection coefficients $\tilde{r}_{p}$ and $\tilde{r}_{\mathrm{s}}$ are given by

$$
\begin{aligned}
\tilde{r}_{p} & =\frac{\sqrt{\tilde{\varepsilon}_{r}-\sin ^{2} \theta_{i}}-\tilde{\varepsilon}_{r} \cos \theta_{i}}{\tilde{\varepsilon}_{r} \cos \theta_{i}+\sqrt{\tilde{\varepsilon}_{r}-\sin ^{2} \theta_{i}}} \\
\tilde{r}_{s} & =\frac{\cos \theta_{i}-\sqrt{\tilde{\varepsilon}_{r}-\sin ^{2} \theta_{i}}}{\cos \theta_{i}+\sqrt{\tilde{\varepsilon}_{r}-\sin ^{2} \theta_{i}}} .
\end{aligned}
$$

${ }^{2}$ Where the subscript "s" stands for senkrecht, a German word meaning perpendicular. 


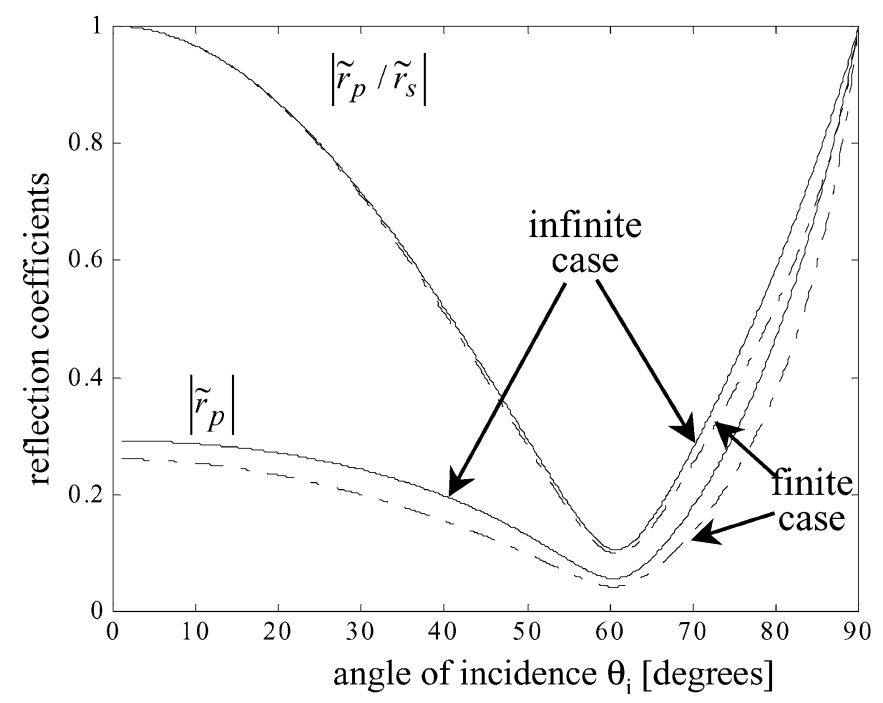

Fig. 3. Theoretical curves of the moduli of the parallel reflection coefficient and the ratio of both reflection coefficients as a function of the angle of incidence in the case of a dielectric sample with finite $(\mathrm{e}=10 \mathrm{~mm})$ or infinite thickness $\left(\varepsilon_{\mathrm{r}}=3-1.0 \mathrm{j}\right)$.

In our measurement configuration, the reflected power, for both polarizations, is recorded by the receiving antenna as a function of the angle of incidence $\theta_{i}$; thus, the corresponding Fresnel coefficients are deduced by subtracting, from this measurement, the power detected when the antennas are placed in the line-ofsight configuration.

\section{B. Methods for the Determination of the Complex Permittivity}

For the estimation of the complex permittivity from the measurement data, we propose several steps which include optimization algorithms for data processing.

1) Choice of the Ratio $\left|\tilde{\mathrm{r}}_{\mathrm{p}} / \tilde{\mathrm{r}}_{\mathrm{s}}\right|^{2}$ : We have theoretically (see Fig. 3) and experimentally observed that, in the case of a finite thickness sample, interference between reflections on the front and back surfaces of the sample induces a fluctuating shape on the curves of each reflection coefficient modulus versus the angle of incidence $\theta_{i}$. On the contrary, the curve of the reflection coefficients ratio modulus does not show such fluctuations and is characterized by a clearly marked minimum: this minimum is useful to determine an initial value of $\tilde{\varepsilon}_{r}$.

2) Choice of the Initial Value of $\tilde{\varepsilon}_{r}$ : Considering different complex permittivity values, we have remarked that the location of the minimum of the ratio $\left|\tilde{\mathrm{r}}_{\mathrm{p}} / \tilde{\mathrm{r}}_{\mathrm{s}}\right|^{2}$ is very close to the pseudo-Brewster's angle $\theta_{B}$, which characterizes the minimum of the curve of the parallel reflection coefficient modulus (see Fig. 3). Moreover, Table I shows that this minimum angle has the same value for infinite and finite thickness samples, respectively. Consequently, we can conclude that the angular position of these minima characterizes accurately the reflection on the first interface. Moreover, the theoretical shapes of the two curves, in the infinite and finite thickness cases, are all the more similar since the contribution of reflection on the back side of the sample can be supposed negligible (the thickness and the losses should be large enough); in such a case, it can be assumed that there is no reflection on the reverse surface of the sample. At
TABLE I

INCIDENCE ANGLES OF THE MINIMUM OF THE RATIO $|\tilde{R}|$ OF THE REFLECTION CoEFFICIENTS AND THE PARALlel ReFlection COEFFICIENT $\left|\tilde{R}_{P}\right|$ $\left(\varepsilon_{r}^{\prime}=3, e=10 \mathrm{~mm}, \mathrm{~F}=10 \mathrm{GHz}, 600\right.$ SAMPLES IN THE INTERVAL $\left.\left[0,90^{\circ}\right]\right)$

\begin{tabular}{c|c|c|c|c|c}
\cline { 2 - 6 } & $\varepsilon_{r}{ }^{\prime \prime}$ & 0 & 1 & 3 & 5 \\
\hline \multirow{2}{*}{ Infinite } & $|\tilde{R}|$ & 59.99 & 60.73 & 64.59 & 68.01 \\
\cline { 2 - 6 } & $\left|\tilde{R}_{p}\right|$ & 59.99 & 60.43 & 63.4 & 66.67 \\
\hline \multirow{2}{*}{ Finite } & $\mid \tilde{R}^{*}$ & 59.99 & 60.73 & 64.59 & 68.01 \\
\cline { 2 - 6 } & $\left|\tilde{R}_{p}\right|$ & 59.99 & 60.43 & 63.4 & 66.67 \\
\hline
\end{tabular}

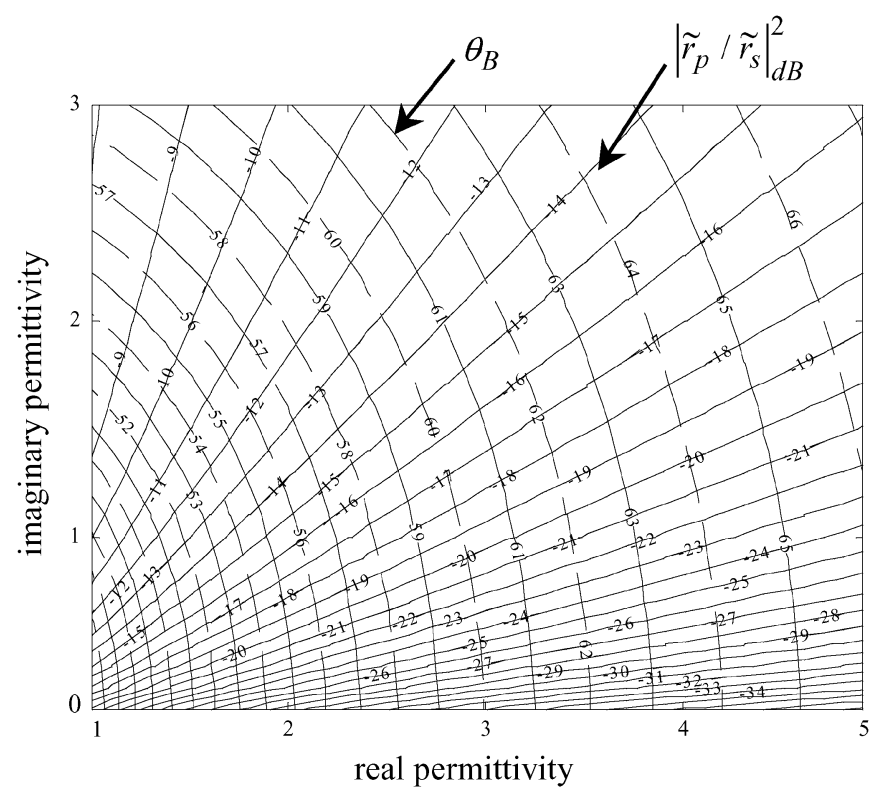

Fig. 4. Chart of constant $\Theta_{B}$ and constant $\left|r_{p} / r_{s}\right|_{(d B)}^{2}$ value lines for the determination of $\tilde{\varepsilon}$ using the Fresnel method in the case of an infinite thickness sample.

last, the position of both curves minima is all the more similar since the relative loss factor value $\varepsilon_{r}^{\prime \prime}$ is small.

We have shown (see Fig. 4) that theoretically, from the determination of the angular position $\theta_{B}$ of the minimum of the reflection coefficients ratio modulus $\left(\theta_{B},\left|\tilde{\mathrm{r}}_{\mathrm{p}} / \tilde{\mathrm{r}}_{\mathrm{s}}\right|^{2}\right)$, an estimate of the complex permittivity $\tilde{\varepsilon}$ could be computed without ambiguity.

3) The Numerical Multistep Method for the Estimation of $\tilde{\varepsilon}_{r}$ : The use of several sets of measurement data obtained at several angles of incidence should obviously yields a statistically relevant estimate of $\tilde{\varepsilon}$, since it allows one to take into account the uncertainties associated with the experimental environment. ${ }^{3}$ In this aim, we have developed the following multistep approach.

1) A first estimate of the complex permittivity $\tilde{\varepsilon}$, say, $\tilde{\varepsilon}_{B}$, is deduced from the coordinates of the minimum of the experimental curve $\left|\tilde{r}_{p} / \tilde{r}_{s}\right|^{2}=F\left(\theta_{i}\right)$ (see Fig. 3).

${ }^{3}$ These uncertainties are caused by the nonuniform radiation patterns of the antennas and also the power and angle measurements. 
2) The value of $\tilde{\varepsilon}_{\mathrm{B}}$ is located on the chart of constant $\theta_{B}$ and constant $\left|\tilde{r}_{\mathrm{p}} / \tilde{r}_{\mathrm{s}}\right|^{2}$ value lines (see Fig. 4).

3) From an estimation of the uncertainties associated with the measurements of $\theta_{B}$ and $\left|\tilde{r}_{\mathrm{p}} / \tilde{r}_{\mathrm{s}}\right|^{2}$, an uncertainty area centered on $\tilde{\varepsilon}_{B}$ is deduced.

4) Several values of $\tilde{\varepsilon}$ are computed by a uniform sampling of $\varepsilon^{\prime}$ and $\varepsilon^{\prime \prime}$ : with each of them, is associated a curve of either $\left|\tilde{r}_{\mathrm{p}} / \tilde{\mathrm{r}}_{\mathrm{s}}\right|^{2}$ or $\left(\left|\tilde{\mathrm{r}}_{\mathrm{p}}\right|^{2}\right.$ and $\left|\tilde{\mathrm{r}}_{\mathrm{s}}\right|^{2}$ jointly) as a function of $\theta_{\mathrm{i}}$.

5) Among these curves, for the one that fits at best (according to a least squares criterion) the measurement data are selected: its corresponding parameter $\tilde{\varepsilon}_{\text {opt }}$ is adopted as the best estimated value for $\tilde{\varepsilon}$.

In the least squares criterion adopted, weighting coefficients were chosen inversely proportional to the value of measurement data. Even if this choice is empirical from a statistical point of view, we have remarked that it allows to enhance the poor numerical conditioning of the problem, induced by the fluctuating characteristics of the measurements. The cost functions $C_{(p, s)}$ and $C_{(p / s)}$, associated, respectively, with the joined parallel and perpendicular reflection coefficients [index $(p, s)]$ and with their ratio (index $(p / s)$ ), are thus defined as

$$
\begin{aligned}
C_{(p, s)}= & \sum_{i=1}^{N} p_{i}^{(p)}\left(\left|\tilde{r}_{t, i}^{(p)}\right|^{2}-\left|\tilde{r}_{m, i}^{(p)}\right|^{2}\right)^{2} \\
& +p_{i}^{(s)}\left(\left|\tilde{r}_{t, i}^{(s)}\right|^{2}-\left|\tilde{r}_{m, i}^{(s)}\right|^{2}\right)^{2} \\
C_{(p / s)}= & \sum_{i=1}^{N} p_{i}\left(\left|\tilde{r}_{t, i}^{(p)} / \tilde{r}_{t, i}^{(s)}\right|^{2}-\left|\tilde{r}_{m, i}^{(p)} / \tilde{r}_{m, i}^{(s)}\right|^{2}\right)^{2}
\end{aligned}
$$

where $\tilde{r}_{\mathrm{t}, \mathrm{i}}^{(\mathrm{p})}$ denotes the $i$ th theoretical (index $t$ ) reflection coefficient in the parallel polarization and $\tilde{r}_{m, i}^{(s)}$ denotes the $i$ th measured (index $m$ ) reflection coefficient in the perpendicular polarization). As the uncertainties on the angle of incidence can be considered as constant, and as these uncertainties were remarked to have negligible influence on the results, we have not considered them in the following developments.

The weighting coefficients were chosen as

$$
\begin{aligned}
p_{i}^{(p, s)} & =\frac{1}{\left|\tilde{r}_{m}^{(p, s)}\right|^{2}} \\
p_{i} & =\frac{1}{\left|\tilde{r}_{m p} / \tilde{r}_{m s}\right|^{2}} .
\end{aligned}
$$

The best estimate of $\tilde{\varepsilon}$ is finally derived by minimizing one of the two following cost functions:

$$
\begin{aligned}
\text { (i) } C_{1}(\theta, r) & =C_{(p, s)} \\
\text { (ii) } C_{2}(\theta, r) & =C_{(p / s)} .
\end{aligned}
$$

\section{THE REFLECTION ElLIPSOMETRY METHOD}

\section{A. General Principles}

Reflection ellipsometry adapted to the microwave frequencies, as first proposed by Stetiu [27], is a technique based on measurements of the change of polarization state of an incident polarized electric field ${ }^{4}$ after its reflection on the air-sample interface [26], for a fixed angle of incidence $\theta_{i}$, as shown in Fig. 1. Because of the difference in the magnitudes and/or the phases of the Fresnel reflection coefficients for the two linear parallel (p) and perpendicular (s) polarizations, the polarization of the electric field generally changes from linear to elliptic [26], [28]-[32]. The knowledge of the ellipse of polarization requires the determination of the ratio $\tilde{\rho}$ of the complex Fresnel reflection coefficients for the (p) and (s) polarizations that depends on the relative complex permittivity $\tilde{\varepsilon}_{r}$ as follows:

$$
\tilde{\rho}=\frac{\tilde{r}_{p}}{\tilde{r}_{s}} .
$$

It is convenient to write $\tilde{\rho}$ in its polar form to express later on the relations of ellipsometry

$$
\tilde{\rho}=\tan \left(\psi_{r}\right) e^{j \Delta_{r}}
$$

where $\Delta_{r}=\delta_{r p}-\delta_{r s}$ is the difference between the phases associated with $\tilde{r}_{p}$ and $\tilde{r}_{s}$ and $\psi_{r}$ represents the modulus of $\tilde{\rho}$.

Thus, $\psi_{r}$ and $\Delta_{r}$ represent the amplitude and phase shifts experienced upon reflection by the parallel and perpendicular components of the electric field.

Supposing that the signal of the detector is a linear function of the received power, the detected power $P_{d}$ is given by

$$
P_{d}=P_{p}+P_{s}+2 \sqrt{P_{p} P_{s}} \cos \left(\Delta_{r}\right)
$$

where $P_{p}$ and $P_{s}$ denote, respectively, the detected powers relative to the two components of the incident electric field $\vec{E}$, as projected on the (p) and (s) polarization axes of the receiving antenna. Thus

$$
\begin{aligned}
& P_{p}=|\vec{E}|^{2}\left|\tilde{r}_{p}\right|^{2} \cos ^{2}(P) \cos ^{2}(A) \\
& P_{s}=|\vec{E}|^{2}\left|\tilde{r}_{s}\right|^{2} \sin ^{2}(P) \sin ^{2}(A)
\end{aligned}
$$

where $P$ and $A$ are the polarization angles of the transmitting and receiving antennas, respectively.

Then, the detected power yields

$$
\begin{array}{r}
P_{d}=P_{0}\left[1-\cos (2 P) \cos \left(2 \psi_{r}\right)+\cos (2 A)\left(\cos (2 P)-\cos \left(2 \psi_{r}\right)\right)\right. \\
\left.+\sin \left(2 \psi_{r}\right) \sin (2 P) \cos \left(\Delta_{r}\right) \sin (2 A)\right] \quad(11)
\end{array}
$$

where the power $P_{0}$, which is the average value of $P_{d}$, writes

$$
P_{0}=\frac{|\vec{E}|^{2}}{4 \cos ^{2} \psi_{r}}\left|\tilde{r}_{s}\right|^{2} .
$$

Without loss of generality, a simplified analytical expression of the reflected power can be considered, namely, when the transmitting antenna polarization angle is chosen as $P= \pm \pi / 4$. In this case, the power of the detected electric field versus the analysis angle $A$ writes as

$P_{d}=P_{0}\left[1-\cos (2 A) \cos \left(2 \psi_{r}\right) \pm \sin (2 A) \sin \left(2 \psi_{r}\right) \cos \left(\Delta_{r}\right)\right]$.

\footnotetext{
${ }^{4}$ Any incident polarized field is a combination of a parallel and a perpendicular wave.
} 


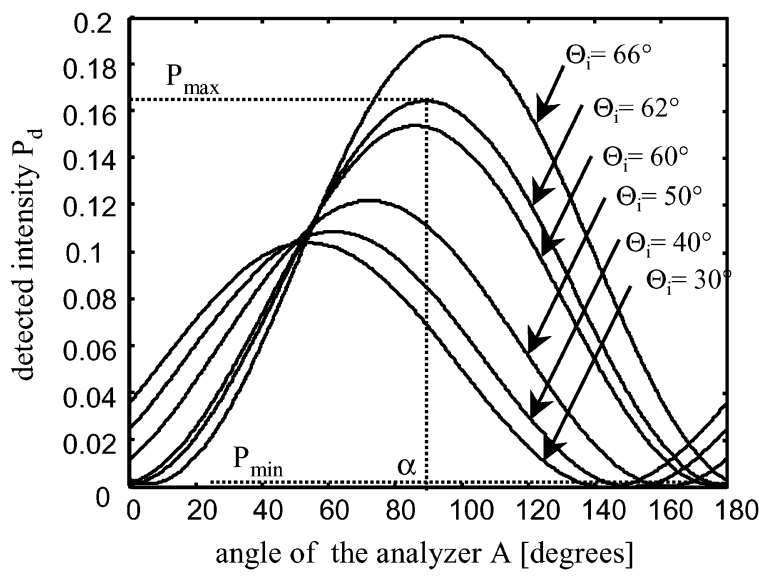

Fig. 5. Variations of the theoretical curves $P_{d}=f(A)$ as a function of the angle of incidence $\theta_{\mathbf{i}}$.

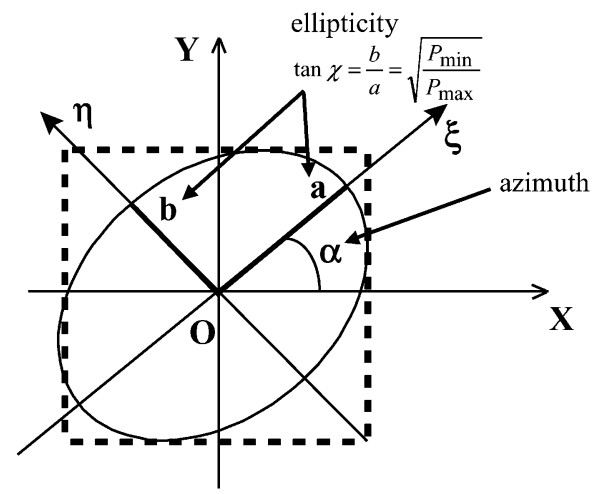

Fig. 6. Ellipse of polarization of the linearly polarized incident electric field after its reflection on the sample surface.

The theoretical variations of $P_{d}$ versus $A$ are sketched on Fig. 5 (in the case of a thick sample with complex permittivity $\tilde{\varepsilon}_{r}=$ $3.5-j$ measured at $10 \mathrm{GHz}$ with different angles of incidence). Two relevant parameters are associated with this curve, namely, the angle $\alpha$ (the azimuth angle of the ellipse) corresponding to the position of the maximal power $P_{\max }$ of the curve and the ratio $\left(P_{\min } / P_{\max }\right)$ corresponding to the extremal values of $P_{d}$.

\section{B. Method for the Determination of the Complex Permittivity}

1) The Analytical Approach: The measured parameters $\alpha$ and $\left(P_{\min } / P_{\max }\right)$ allow one to retrieve the complex permittivity according to the following steps.

1) The parameter $\chi$ is related to the ratio $\left(P_{\min } / P_{\max }\right)$, according to

$$
\tan \chi=\sqrt{\frac{P_{\min }}{P_{\max }}}
$$

We remark that $\tan \chi$ is also related to the ellipticity (see Fig. 6) according to

$$
\tan \chi=\frac{b}{a}
$$

2) The parameters $\psi_{r}$ and $\Delta_{r}$ can be deduced from the angles $\alpha$ and $\chi$ according to the two fundamental relations of ellipsometry

$$
\begin{aligned}
\tan 2 \alpha & =\tan 2 \psi_{r} \cos \Delta_{r} \\
\sin 2 \chi & =\sin 2 \psi_{r} \sin \Delta_{r} .
\end{aligned}
$$

3) Thus, the ratio $\left(\tilde{r}_{p} / \tilde{r}_{s}\right)$ can be determined and, in turn, the value of the relative complex permittivity $\tilde{\varepsilon}_{\text {r }}$ as implicitly expressed by (2).

Two remarks are of interest at this point.

1) In the case of a single-layer sample, the preceding steps can be formulated by a simple analytic relationship between $\tilde{\varepsilon}_{\mathrm{r}}$ and $\left(\alpha, P_{\min } / P_{\max }\right)$. However, we have used a graphical approach, based on constant- $\alpha$ and constant $-\left(P_{\min } / P_{\max }\right)$ curves in the $\left(\varepsilon_{r}^{\prime}, \varepsilon_{r}^{\prime \prime}\right)$ plane, that allows visualization of the influence of measurement uncertainties on the estimated value of the complex permittivity (see Fig. 7).

2) Our method deals only with moduli of the detected power of the electric field as a function of the analyzer angle $A$. Nevertheless, it allows one to estimate both modulus and phase of the ratio $\tilde{r}_{p} / \tilde{r}_{s}$ without having to vary the angle of incidence $\theta_{\mathrm{i}}$, as opposed to the Fresnel method.

2) The Numerical Approach: Having characterized the variation laws of the fundamental parameters extracted from the ellipsometric curve, we propose a numerical approach for the determination of the complex permittivity of a single-layer sample.

It consists of the following steps.

1) The measured values of $\left(P_{\max }\right)_{\mathrm{dB}}$ and $(\alpha)_{\text {degrees }}$ and a contour lines chart are used to provide a first guess $\tilde{\varepsilon}_{r 0}=$ $\left(\varepsilon_{r 0}^{\prime}, \varepsilon_{r 0}^{\prime \prime}\right)$ of the permittivities [see Fig. 7(a)].

2) The minimum value $P_{\min }$ of the theoretical curve $P_{d}=$ $f(A)$ associated with $\tilde{\varepsilon}_{r 0}$ is extracted.

3) The value $\tilde{\varepsilon}_{r 0}$ is located on constant- $\left(P_{\min } / P_{\max }\right)_{\mathrm{dB}}$ and constant- $(\alpha)_{\text {degrees }}$ contour line charts [see Fig. 7(b)]. Then, couples of real and imaginary permittivities $\left(\varepsilon_{r}^{\prime}, \varepsilon_{r}^{\prime \prime}\right)$ are regularly sampled in the parallelepipedic uncertainty area centered on $\tilde{\varepsilon}_{r 0}$ (see Fig. 8).

4) With each point in this area, a corresponding characteristic $P_{d}=f(A)$ is associated.

5) The best characteristic is retained as the one fitting best the experimental data points, according to a least squares criterion.

6) Optimal values $\left(\varepsilon_{r_{\text {opt }}}^{\prime}, \varepsilon_{r_{\text {opt }}}^{\prime \prime}\right)$ are inferred as the couple corresponding to this best characteristic.

For example, the graphical device of Fig. 8 allows one to evaluate the incidence, on the estimation of the complex permittivity, of absolute uncertainties of $\pm 0.4 \mathrm{~dB}$ on the measurement of $\left(P_{\min } / P_{\max }\right)_{\mathrm{dB}}$ and of $\Delta \alpha= \pm 0.5^{\circ}$ on the knowledge of $\alpha$, these values being compatible with the accuracy of our experimental setup. Fig. 8 shows that all possible values of the complex permittivity are located inside a parallelogram such that, in this particular case of a thick sample with permittivity $\tilde{\varepsilon}_{r}=3.5-j$, the uncertainties can be read as $2 \Delta \varepsilon_{r}^{\prime}=0.39$ and $2 \Delta \varepsilon_{r}^{\prime \prime}=0.35\left(\theta_{i}=45^{\circ}\right)$. 


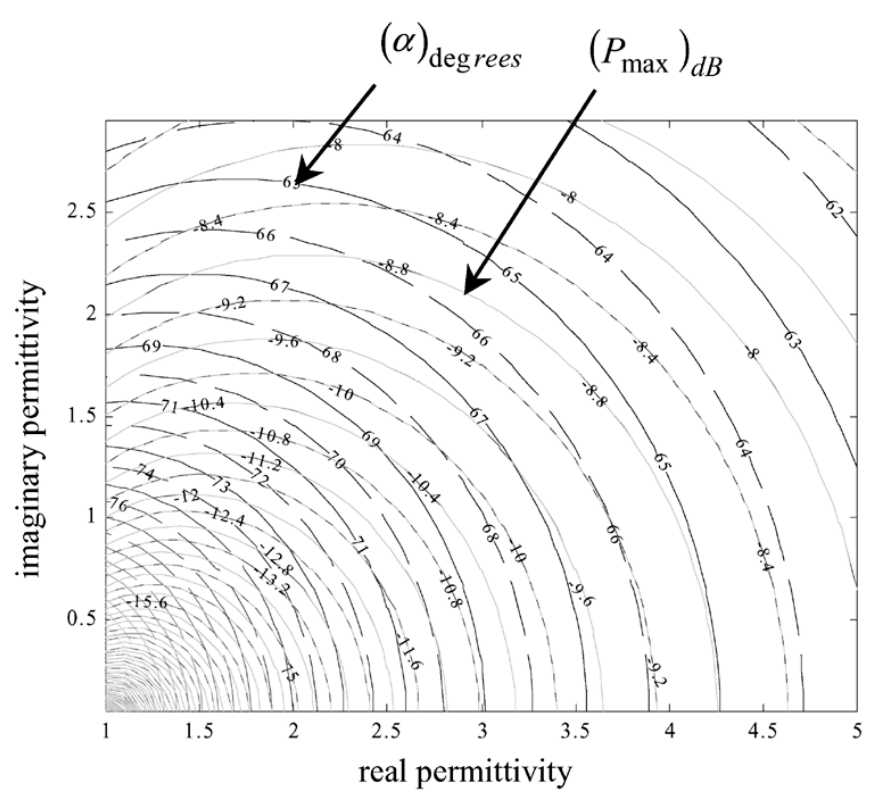

(a)

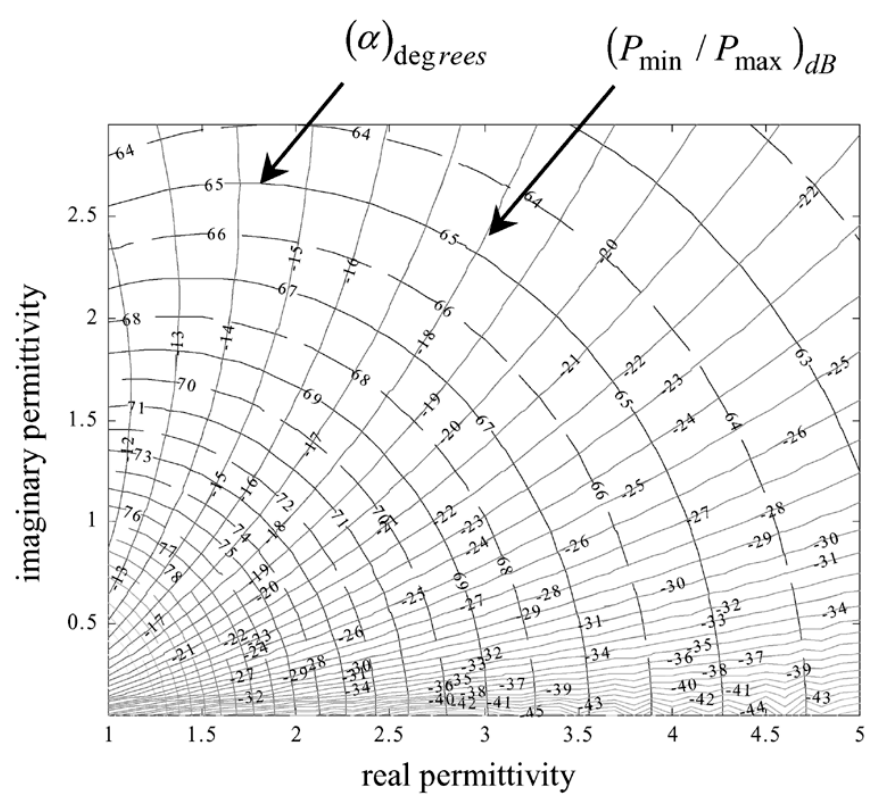

(b)

Fig. 7. Charts of (a) constant- $(\alpha)_{\text {degrees }}$, constant- $\left(\mathrm{P}_{\max }\right)_{\mathrm{dB}}$ and (b) constant $-\left(\mathrm{P}_{\min } / \mathrm{P}_{\max }\right)_{\mathrm{dB}}$ value lines for the determination of $\tilde{\varepsilon}$ using ellipsometry in the case of an infinite thickness sample $\left(\Theta_{\mathrm{i}}=45^{\circ}\right)$.

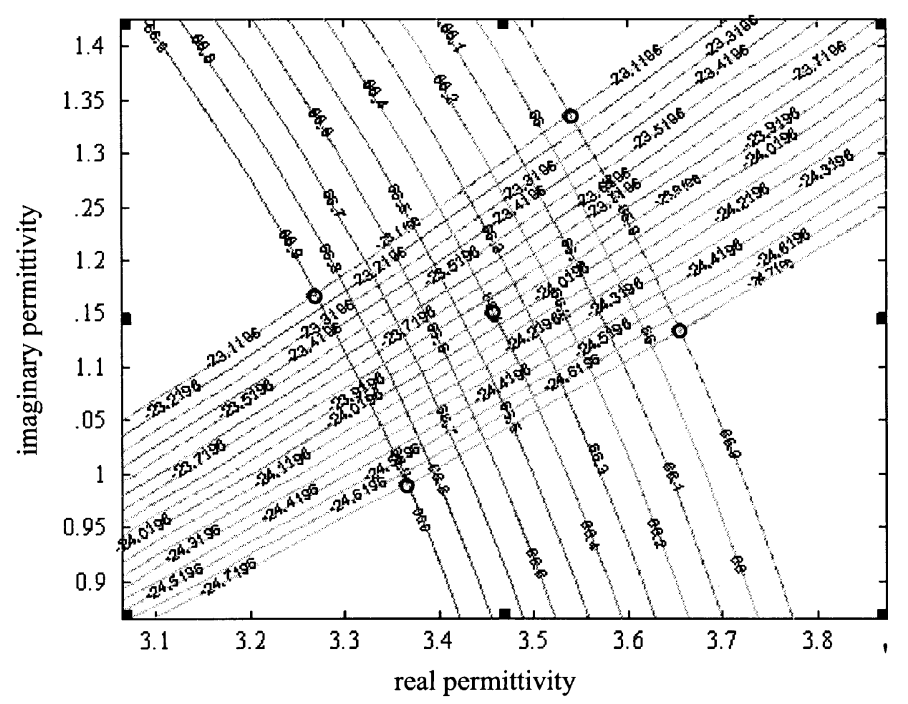

Fig. 8. Parallelogram of uncertainties with constant- $(\alpha)_{\text {degrees }}$ and constant $-\left(\mathrm{P}_{\min } / \mathrm{P}_{\max }\right)_{\mathrm{dB}}$ curves centered on the complex permittivity $\tilde{\varepsilon}_{r}=3.5-j$ in the case of an infinite thickness sample $\left(\Theta_{\mathrm{i}}=45^{\circ}\right)$.

\section{PARAmeters Study FOR REFLECTION ElLIPSOMETRY}

In order to characterize more precisely the sensitivity of our approach, we propose now to take into account explicitly the influence of some parameters of interest-namely, the angle of incidence $\theta_{i}$, the measured power ratio $\left(P_{\min } / P_{\max }\right)$, and the maximum polarization angle $\alpha$ - on the estimation of the complex permittivity $\tilde{\varepsilon}$.

\section{A. Optimal Choice for $\theta_{i}$}

One of the major advantages of our approach is that the angle of incidence $\theta_{i}$ is fixed all along the measurement process. However, its value can be chosen optimally in order to minimize the uncertainty on the estimation of the complex permittivity.
Fig. 5 shows measured parameters $\alpha$ and $P_{\max }$ as function of the angle of incidence $\theta_{i}$, in the case of a thick sample with $\tilde{\varepsilon}_{r}=3.5-j-$ a rough estimate of the permittivity of the concrete wall we have studied. It appears from this figure that the value of $\alpha$ grows with the angle of incidence $\theta_{i}$. Theoretical analyses show clearly that the highest sensitivity to these parameters, inducing the best accuracy of the method, is achieved for $\theta_{i}$ varying in the range $\left[35^{\circ}, 50^{\circ}\right]$. We have checked moreover that this result holds on a large range of complex permittivity values.

\section{B. Influence of $\alpha$ and $P_{\min } / P_{\max }$ on $\varepsilon_{r}^{\prime}$}

For a given relative loss factor $\varepsilon_{r}^{\prime \prime}$ and an angle of incidence $\theta_{i}=40^{\circ}$, the curves $P_{d}=f(A)$ show that the angle $\alpha$ and 
the ratio $\left(P_{\min } / P_{\max }\right)$ decrease with the real permittivity. Considering the maximal measurement uncertainties $( \pm 0.4 \mathrm{~dB}$ for powers $P_{d}$ and $\pm 0.5^{\circ}$ for the polarization angles $A$ ), the relative variations on the value of $\varepsilon_{r}^{\prime}$ caused by $\Delta P_{\max }= \pm 0.4 \mathrm{~dB}$ and $\Delta \alpha= \pm 0.5^{\circ}$ have been calculated for two real permittivities. Then, considering $\varepsilon_{r}^{\prime}=2.5$ (respectively, 5) for $\varepsilon_{r}^{\prime \prime}=1$, the relative variations induced by $\Delta P_{\max }$ are $\Delta \varepsilon_{r}^{\prime} / \varepsilon_{r}^{\prime}= \pm 8.5 \%$ (respectively, 9.2\%) and the relative variations induced by $\Delta \alpha$ are $\Delta \varepsilon_{r}^{\prime} / \varepsilon_{r}^{\prime}= \pm 6.45 \%$ (respectively, $7.4 \%$ ). We remark that the major source of uncertainties is the measurement of $P_{\max }$.

\section{Influence of $\alpha$ and $P_{\min } / P_{\max }$ on $\varepsilon_{r}^{\prime \prime}$}

For a given relative real part of the permittivity and an angle of incidence $\theta_{i}=40^{\circ}$, the curves $P_{d}=f(A)$ show that, contrary to the ratio $\left(P_{\min } / P_{\max }\right)$, angle $\alpha$ decreases with the relative loss factor. Considering the same measurement uncertainties on $\alpha$ and $P_{\max }$ as in Section IV-B, the relative variations $\left(\Delta \varepsilon_{r}^{\prime \prime} / \varepsilon_{r}^{\prime \prime}\right)$ have been calculated for two relative loss factors. Then, considering $\varepsilon_{r}^{\prime \prime}=2.5$ (respectively, 5) for $\varepsilon_{r}^{\prime}=3.5$, the relative variations induced by $\Delta P_{\max }$ are $\Delta \varepsilon^{\prime \prime} / \varepsilon^{\prime \prime}= \pm 16.1 \%$ (respectively, 13.2\%) and the relative variations induced by $\Delta \alpha$ are $\Delta \varepsilon^{\prime \prime} / \varepsilon^{\prime \prime}= \pm 13.2 \%$ (respectively, 9.36\%). It appears that the determination of the relative loss factor is more sensitive to measurement uncertainties on $P_{\max }$ and $\alpha$ when its value is less than one.

\section{Conclusion And Perspectives}

Reflection ellipsometry has been proposed as an alternative low-cost technique for the in situ characterization of a dielectric material at microwave frequencies; the objective is to use the setup in indoor environments for the characterization of construction walls. In the first part of this paper, theoretical developments have been made to optimize the experimental conditions and thus to determine the complex permittivity of a single-layer sample (the thickness is supposed to be known) from experimental data using a graphical approach. This determination is based on an original numerical multistep method using contour line charts, which allows studying the influence of measurement uncertainties associated to the relevant parameters. This leads to a software tool (COTREMO) which requires no theoretical knowledge from the user, since the algorithmic process is proposed as a self-contained and robust toolbox.

We propose to further compare, in the experimental part of this paper (Part II), the performance of this technique with the performance of the classical Fresnel approach. For this aim, we have also developed a specific numerical multistep method, based also on contour line charts, for the estimation of the complex permittivity.

From this point, future trends on ellipsometry would include the characterization of other types of materials, such as doublelayer samples, and the study of the transmission configuration.

\section{ACKNOWLEDGMENT}

The authors would like to thank Prof. M. Sylvain for his suggestions relative to physical developments and the reviewers for helpful comments.

\section{REFERENCES}

[1] M. N. Afsar, J. R. Birch, and R. N. Clarke, "The measurement of the properties of materials," Proc. IEEE, vol. 74, pp. 183-199, 1986.

[2] J. Musil and F. Zacek, Microwave Measurements of Complex Permittivity by Free-Space Methods and Their Applications. New York: Elsevier, 1986.

[3] E. Nyfors and P. Vainikainen, Industrial Microwave Sensors: Artech House, 1989.

[4] I. L. Al-Qadi, D. K. Ghodgaonkar, and V. K. Varadan, "Effect of moisture on asphaltic concrete at microwave frequencies," IEEE Trans. Geosci. Remote Sensing, vol. 29, no. 5, pp. 710-717, May 1991.

[5] T. Hauschild and F. Menke, "Moisture measurement in masonry walls using noninvasive reflectometer," Electron. Lett., vol. 34, no. 25, pp. 2413-2414, 1998.

[6] J. G. Wilson and H. W. Whittington, "Variations in the electrical properties of concrete with change in frequency," Proc. Inst. Elect. Eng., pt. A, vol. 137, no. 5, pp. 246-254, 1990.

[7] J. Curtis and R. Narayanan, "Effects of laboratory procedures on soil electrical property measurements," IEEE Trans. Instrum. Meas., vol. 47, no. 6, pp. 1474-1480, Dec. 1998.

[8] W. H. Honcharenko, H. L. Bertoni, J. L. Dailing, J. Qian, and H. D. Yee, "Mechanisms governing UHF propagation on single floors in modern office buildings," IEEE Trans. Veh. Technol., vol. 41, no. 4, pp. 496-504, 1992.

[9] J. H. Tarng, W. R. Chang, and B. J. Hsu, "Three-dimensional modeling of 900-MHz and 2.44-GHz radio propagation in corridors," IEEE Trans. Veh. Technol., vol. 46, no. 2, pp. 519-527, Mar. 1997.

[10] K. R. Rizk, J. F. Wagen, and F. Gardiol, "Two-dimensional ray-tracing modeling for propagation prediction in microcellular environments," IEEE Trans. Veh. Technol., vol. 46, no. 2, pp. 508-517, Mar. 1997.

[11] K. Sato, H. Kozima, H. Masuzawa, T. Manabe, T. Ihara, Y. Kasashima, and K. Yamaki, "Measurements of reflection characteristics and refractive indices of interior construction materials in millimeter-wave bands," in IEEE 45th Veh. Technol. Conf., vol. 1, 1995, pp. 449-453.

[12] G. L. Friedsam and E. M. Biebl, "A broadband free-space dielectric properties measurement system at millimeter wavelengths," IEEE Trans. Instrum. Meas., vol. 46, no. 2, pp. 515-518, Apr. 1997.

[13] R. G. Nitsche, J. Preissner, and E. M. Biebl, "A free-space technique for measuring the complex permittivity and permeability in the millimeter wave range," in IEEE Microwave Theory Tech. Dig., 1994, pp. $1465-468$.

[14] A. C. Lynch, H. D. Griffiths, S. Appleton, A. L. Cullen, A. Khosrowbeygi, and R. Benjamin, "Free-wave measurement of permeability and permittivity of ferrites at millimeter-wave frequencies," Proc. Inst. Elect. Eng. Sci. Meas. Technol., vol. 142, no. 2, pp. 169-175, 1995.

[15] C. F. Yang, C. J. Ko, and B. C. Wu, "A free-space approach for extracting the equivalent dielectric constants of the walls in buildings," in Antennas Propagat. Soc. Int. Symp., vol. 2, 1996, pp. 1036-1039.

[16] J. P. Pugliese, A. Hammoudeh, and M. O. Al-Nuaimi, "Reflection and transmission characteristics of building materials at $62 \mathrm{GHz}$," Inst. Elect. Eng. Colloq. Radio Communication Microwave Millimeter Wave Frequencies, pp. 6/1-6, 1996.

[17] K. Sato, T. Manabe, J. Polivka, T. Ihara, Y. Kasashima, and K. Yamaki, "Measurement of the complex refractive index of concrete at $57.5 \mathrm{GHz}$," IEEE Trans. Antennas Propag., vol. 44, no. 1, pp. 35-40, 1996.

[18] K. Sato, T. Manabe, T. Ihara, H. Saito, S. Ito, T. Tanaka, K. Sugai, N. Ohmi, Y. Murakami, M. Shibayama, Y. Konishi, and T. Kimura, "Measurements of reflection and transmission characteristics of interior structures of office building in the $60 \mathrm{GHz}$ band," IEEE Trans. Antennas Propag., vol. 45, no. 12, pp. 1783-1791, Dec. 1997.

[19] O. Landron, M. J. Feuerstein, and T. S. Rappaport, "In situ microwave reflection coefficient measurements for smooth and rough exterior wall surfaces," in 43th Conf. Veh. Technol., 1993, pp. 77-80.

[20] O. Landron, M. J. Feuerstein, and T. S. Rappaport, "A comparison of theoretical and empirical reflection coefficients for typical exterior wall surfaces in a mobile radio environment," IEEE Trans. Antennas Propag., vol. 44, no. 3, pp. 341-351, Mar. 1996.

[21] Y. Huang and M. Nakhkash, "Characterization of layered dielectric medium using reflection coefficient," Electron. Lett., vol. 34, no. 12, pp. 1207-1208, 1998.

[22] M. Nakhkash, Y. Huang, and M.T.C. Fang, "A new free-space technique for measuring the complex permittivity and thickness of materials," in Inst. Elect. Eng. Nat. Conf. Antennas Propag., 1999, pp. 23-26. 
[23] D. K. Ghodgaonkar, V. V. Varadan, and V. K. Varadan, "Free-space measurement of complex permittivity and complex permeability of magnetic materials at microwave frequencies," IEEE Trans. Instrum. Meas., vol. 39, no. 2, pp. 387-394, Apr. 1990.

[24] M. H. Umari, D. K. Ghodgaonkar, and V. V. Varadan, "A free-space bistatic calibration technique for the measurement of parallel and perpendicular reflection coefficients of planar samples," IEEE Trans. Instrum. Meas., vol. 40, no. 1, pp. 19-24, Feb. 1991.

[25] M. Born and E. Wolf, Principles of Optics, 6th ed. New York: Pergamon, 1980.

[26] R. M. A. Azzam and N. M. Bashara, Ellipsometry and Polarized Light. New York: Elsevier, 1999.

[27] P. Stetiu and B. Hannover, "Ellipsométrie en microondes," Journées Caractérization Microondes et Matériaux, pp. 281-284, Mar. 2000.

[28] V. A. Konev, S. A. Tikhanovich, and M. I. Zhigalko, "Error in the determination of the dielectric properties and thickness of surface layers by the method of radiometric ellipsometry," Sov. J. Nondest. Test. (US), vol. 22 , no. 6, pp. 363-366, Jun. 1986.

[29] J. Lekner, "Determination of complex refractive index and thickness of a homogeneous layer by combined reflection and transmission ellipsometry," J. Opt. Soc. Amer. A, vol. 11, no. 7, pp. 2156-2158, 1994.

[30] G. Bader, P. V. Ashrit, F. E. Girouard, and V. V. Truong, "Reflectiontransmission photoellipsometry: Theory and experiments," Appl. Opt., vol. 34, no. 10, pp. 1684-1691, 1995.

[31] R. Radoi and C. Flueraru, "Determination of the best incidence angle for ellipsometric measurements," in 21st Int. Semiconductor Conf. (CAS'98), Sinaia, Romania, 1998, pp. 245-248.

[32] M. H. Chiu, J. Y. Lee, and D. C. Su, "Complex refractive-index measurement based on Fresnel's equations and the uses of heterodyne interferometry," Appl. Opt., vol. 38, no. 19, pp. 4047-4052, 1999.

[33] F. Sagnard, "Determination of complex permittivity and thickness of a single-layer material using reflection ellipsometry at several angles of incidence," Microw. Opt. Technol. Lett., vol. 36, no. 40, pp. 243-248, Feb. 2003.

[34] F. Sagnard, F. Bentabet, and C. Vignat, "In situ measurements of the complex permittivity of materials using reflection ellipsometry in the microwave band: Experiments (Part II)," IEEE Trans. Instrum. Meas., vol. 54, no. 3, pp. 1274-1282, 2005.

[35] A. H. Shivola, "Self-consistency aspects of dielectric mixing theories," IEEE Trans. Geosci. Remote Sens., vol. 27, no. 4, pp. 710-717, 1989.

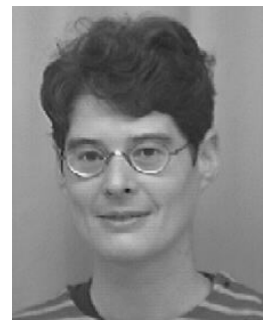

Florence Sagnard was born in Paris, France, in 1965. She received the engineer diploma and the D.E.A. degree in electronics from Université Pierre et Marie Curie, France, in 1990 and the Ph.D. degree from Université d'Orsay, France, in 1996.

From 1990 to 1993, she was an Engineer on military electronic systems. From 1993 to 1996 , she was with CEA and ONERA. Since 1997, she has been an Assistant Professor at Université de Marne-La-Vallée, France. Her research interests include microwave characterization of materials. In September 2002, she joined IETR laboratory of INSA Rennes to devote her work to research on microwave and millimeter propagation inside buildings.

Faroudja Bentabet was born in France in 1978. She received the B.S.C. degree from Université de Marne-la-Vallée, France, in 2000 and the Ph.D. degree in mobile communications from Université de Versailles-Saint-Quentin, France, in 2001 .

She is currently working in the telecommunication industry.

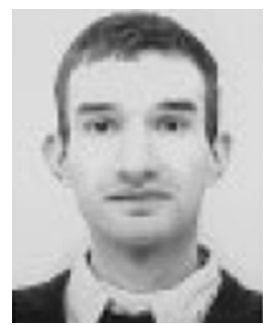

Christophe Vignat was born in France in 1965. He received the B.E.E. degree from Ecole Supérieure d'Electricité, Paris, France, and the Ph.D. degree in physics from Laboratoire des Signaux et Systèmes, Université de Paris-Sud, Orsay, France, in 1993.

Since 1995, he has been an Associate Professor at Université de Marne-La-Vallée, France. After working on adaptive systems, his interests shifted toward information theory and numerical methods applied to physics. 